\title{
Behavioral effects arising from the neural substrates for atypical planning and execution of word production in stuttering
}

\author{
Peter Howella \\ a University College London, Gower St., London WC1E 6BT
}

\begin{abstract}
This article reports on an fMRI study that examined the neural bases of atypical planning and execution processes involved in stuttering (Lu et al., 2010). In the study, twelve stuttering speakers and 12 controls named pictures which required single-syllable, multi-syllable, or repeated-syllable word responses, in the scanner. The factors associated with planning and execution were: 1) number of syllable-sized motor programs; and 2) syllable size and onset complexity. Structural equation modeling revealed two parallel neural circuits (the basal gangliainferior frontal gyrus, premotor area circuit and the cerebellum-premotor area circuit). These were involved in atypical planning and execution processes in stuttering, respectively. The interface between planning and execution in stuttering involved the angular gyrus. This article discusses the relevance of these findings to behavioral theories that also propose separate planning and execution mechanisms behind stuttering.
\end{abstract}

\section{Keywords}

Developmental stuttering; Planning; Execution; Connectivity; EXPLAN

\section{Introduction}

A number of symptoms occur in the speech of speakers who stutter. Those which Johnson et al. (1959) listed were: 1) Incomplete phrases (sometimes called abandonments); 2) Revisions (change of one word for another); 3) Interjections (word and non-word fillers); 4) Whole-word repetitions ("I, I, I"; 5) Phrase repetitions ("in the, in the morning"); 6) Partword repetitions ("k-k-Katy"); 7) Prolongations ("sssister"); and 8) Broken words ("dinosaur").

A threshold is often used to designate how frequently symptoms in these classes occur for a person to be considered as someone who stutters. For example, Yairi and Ambrose (2005) stipulated 3\% of words should have a subset of these symptoms. The designation of a person as someone who stutters depends not only on what symptoms are considered characteristic of stuttering and how frequently they occur, but also on the representativeness of the speech samples on which the assessment is based and the procedures used to count stuttering symptoms (for example, based on informal listening versus stringent computer-based assessments).

Certain variables immediately improve the fluency of people who stutter. Examples are where speakers speech output is delayed or shifted in frequency before they hear it, when

Address correspondence to: Peter Howell, Division of Psychology and Language Sciences and Centre for Human Communications, University College London, Gower Street, London WC1E 6BT, England. p.howell@ucl.ac.uk. 
vocal output is masked or where speakers speak along with others (choral speech). Singing is another form of altered voice control that improves fluency, but this is not used in treatments or experiments as many people feel embarrassed when asked to sing. All these manipulations only last as long as the manipulation is made. There is no evidence that the effects wear off over time. However, some users of prosthetic devices that make some of these manipulations have suggested that this might be the case. This may arise because speakers reduce voice level when they have used the device for some time to reduce the effort involved when speaking with the alteration. This would also reduce the effect of the manipulation on fluency. This needs to be checked as, if it is true, it is possible to neutralize the effect of speaking quietly on fluency by using automatic gain control circuits (Howell, forthcoming).

Some of these variables have been used as components in treatment programs because of their immediate effect on fluency. As noted earlier, the effects are mainly limited to the period whilst the variation occurs whereas traditional treatment interventions do not have immediate effects but are considered to have longer term remedial effects on stuttering in some individuals. The immediacy of the fluency-inducing variables makes them useful in scanning studies when, for instance, investigators want to establish what CNS-changes occur between when a person who stutters is fluent and disfluent.

Biological factors appear to underpin the disorder. For example, linkage and, more recently, association analysis procedures have established a relationship between genes and stuttering. Two recent studies using association analysis have appeared (Lan et al., 2009; Kang et al., 2010). These studies implicated dopaminergic (release controlled through basal ganglia) and lysosomal trafficking genes respectively. In all cases, the mutant genes occurred at significantly higher rates in the stuttering samples than in the non-stuttering control samples.

In the case of stuttering, a lot of work remains to be done to show how genes affect stuttering. First and foremost, independent replications of the genetic findings are required. If replicated, the way that the mutant genes lead to brain disorder that then affects speech production needs to be documented. The work required in tracing this route for another genetic disorder that affects speech production was summarized recently by Dworzynski (forthcoming). She described the speech disorder that affects the KE family, how this shows a simple Mendelian pattern of inheritance and how the gene responsible (FOXP2) was identified. This is the stage where association work with stuttering appears to be at present (subject to replication of the studies). It is already known that the situation with stuttering is more complex than in the case of the KE family (non Mendelian inheritance pattern, polygenic disorder and incomplete penetrance).

The next tasks if the procedures are followed that were employed with the KE family, will involve developing mutant strains of animals where the genes are knocked out (Groszer et al., 2008) or modified (Haesler et al., 2007) so the effects on brain structures and vocalization functions can be established. Again see Dworzynski, (forthcoming) for a review which discusses the relevance of these findings and procedures to stuttering).

Although there is a gap in knowledge between genes and the physiological processes that lie behind stuttering, research has examined how physiological processes and behavioral findings link together in the case of stuttering (Alm, 2004; Howell, 2004). Alm (2004) observed that a sizable proportion of children with developmental stuttering reported closed head injury and he speculated that this might have resulted in basal ganglia damage. Howell (2004) proposed that stuttering is a result of malfunction in the process by which language and motor levels interact and his model accounted for how stuttering symptoms arise and how they change over development (i.e. as a child changes from a person with a good 
chance of recovery into one with less chance of recovery). The model also proposed what were the likely neural substrates of the language, motor and interaction levels and accounted for how the interventions mentioned earlier as being effective and immediate ways of influencing fluency, arose through the operation of these neural mechanisms.

The brain processes and connectivity patterns between them have been assessed for the Howell $(2004 ; 2010)$ model by Lu et al. (2010). Specifically, scanning work has been conducted to test the proposed correspondence between the behavioral findings and the neural substrates that subserve them (Lu et al., 2010). A review of the essential behavioral background to this experimental framework is given. Then Lu et al.'s (2010) work is examined to see how it has advanced understanding about stuttering and the interface between planning and execution. Finally some possible new directions for research are suggested.

\section{Background to the EXPLAN model that involves planning, execution and interface components}

Language planning and speech motor programming and execution are both widely regarded as being affected in people who stutter. Usually either planning or execution processes are considered as being involved. However recent models include both processes (Howell, 2004; Howell, 2007; Howell and Au-Yeung, 2002). Lu et al.'s (2010) work follows this trend and extends it by examining the neural substrates of planning and execution.

According to Howell's theory, some stuttering symptoms are related to the planning processes and some are associated with execution processes as indicated by the different roles each symptom class plays with respect to dealing with disfluency. In particular, wholeword repetitions (and also pauses) are ways of stalling motorically (repeatedly executing a previously generated program) on material prior to other material that is difficult to plan) whereas prolongations part-word repetitions and word breaks reflect planning problems (the repetition, prolongation and hesitation within words signify that the plan was not right or was only partly prepared). Prolongations, part-word repetitions and word breaks are referred to as advancing to indicate the speaker has moved forward prematurely in the speech stream and to contrast with what happens in stalling. The theory is called EXPLAN to acknowledge the contributions of motor execution and language planning. This perspective gives wholeword repetitions a different role (motor based) to symptoms that reflect planning problems. Although this theory focuses on language and motor processes, it should be noted that stuttering affects other things too (e.g. socio-emotional and non-speech cognitive behavior).

Part of the motivation for developing EXPLAN theory was the fact that neither language nor motor processes alone can exclusively account for why stuttering starts in children and why it persists in children who are still stuttering at teenage. No milestones in language development have been identified that explain why stuttering starts (Howell, forthcoming). For instance, the average age of stuttering onset is about three years of age (Yairi and Ambrose, 2005), by which time most syntactic development is complete (Limber, 1973). The subsequent course of stuttering shows no marked problem in other language planning processes. For instance, children who continue to stutter do not exhibit dysarthria, oral dyspraxia, or other signs of an incorrect mental model of the utterance (Brown et al., 2005). The speakers who persist do not show any major abnormality of the speech motor system (motor cortex, basal ganglia, lower motor neurons), as there is no oral weakness, spasticity, tremor, or hypophonia (Brown et al., 2005).

The distinction between language and motor levels in EXPLAN allowed planning (e.g. language processing deficits) and/or execution (motor timing deficits) problems to result in stuttering. Although the language and motor levels are distinct, they have to link together 
(interface) for meaningful speech to be output. According to EXPLAN, the interface accounts for how failures of planning or execution can result in stuttering. The way the model operates is illustrated with the utterance "I split it". The speaker starts by planning "I" which is a simple word. "I" is a word from the function word class and words from this class are, generally speaking, easy to plan so they are generated rapidly. Once the plan is ready, it can be sent to the motor execution processes where the motor programme can also be generated rapidly after which this word can be output. Planning and execution are independent in the model, so whilst motor preparation for this word is taking place, planning for the verb "split" can be performed. "Split" would be more difficult to plan than "I". "Split" is a content word and this class of word is, generally speaking, harder to plan and takes more time to prepare than function words. In this particular case, the difficulty arises because the verb has a consonant cluster and starts with the phone /s/ that is amongst the latest sounds that a child learns. Since "I" can be output rapidly whilst planning for "split" can take some time, the plan for the latter may not be ready at the time that execution of "I" is completed. When this occurs, the plan for "split" is incomplete after "I" has been uttered.

Consequently, the entire next plan cannot be transferred to execution via the interface. The execution processes can either be stopped (paused) or the speaker can repeat the current plans of words that were just received (this results in whole-word or phrase repetition depending how many plans for prior function words are available). Planning can continue and once the complete plan is available, speech can proceed. When the process is stalled because the plan is not available, this results in disfluencies like "I I split it" or "I [pause] split it". The process that leads to stalling is illustrated diagrammatically at the top of Figure 1 when single whole-word repetition occurs. The theory predicts, as observed, that repetitions of function words after the verb will not occur (i.e. "I split it it") because their repetition would not affect planning of the verb.

Children who stutter, like fluent children, begin by stalling as just described when they encounter problems generating difficult items like "split". If, however, the stuttering persists, symptom types change: Speakers then start to produce disfluencies on content words and these are different from stallings. Examples often seen in persistent developmental stuttering are "I sssplit it", "I s.s.split it" and "I s/plit it" (where / indicates a break within the word. According to EXPLAN, these all occur because these speakers have stopped stalling and attempt the word on the basis of an incomplete plan. The plan is generated left to right which explains two things: 1) why these symptoms of stuttering tend to occur at the start of words; 2) that the strategy is not entirely inappropriate as it will work sometimes (when the remainder of the plan arrives during the time the speaker executes the first part). The process that leads to advancing is illustrated diagrammatically at the bottom of Figure 1.

EXPLAN proposed a particular neural substrate and process for execution and the way it communicates with the interface that allowed the change from stallings to advancings (difficulty on the subsequent word) to occur, that also explained why, once this change happens, it is difficult to reverse (i.e. why recovery is unlikely in children who persist in their stuttering beyond teenage).

First, it was proposed that the neural mechanism responsible for execution is the cerebellum (Howell, 2002). This site was proposed primarily because stuttering involves disruption to speech timing that is controlled by the cerebellum. The cerebellum is also traditionally regarded as being involved in sensory-motor integration and such processes have been implicated in stuttering by some authors. There is also evidence for the view that cerebellar operation is different in people who stutter and controls: Children who stutter show performance deficits in the Wing-Kristofferson (1973) isochronous tapping task that work in 
patient with cerebellar damage has linked to the cerebellum (Ivry, 1997). Work has also shown that children who stutter perform worse than controls on the Dow-Moruzzi battery of tasks that assess cerebellar performance (Howell, Davis and Williams, 2008). Other evidence with fluent speakers suggest that delaying voice feedback disrupts cerebellar processes (Howell and Sackin, 2002) and this may explain the positive effects of this manipulation on the fluency of people who stutter. The cerebellum has also been implicated in feedforward accounts of motor control such as that of Kawato, Furakawa and Suzuki (1987). In feedforward control speakers predict the consequences of an action and subsequently check whether the action fits with the prediction. Feedforward processing was used in modified form in EXPLAN to account for how speakers detect whether the plan for speech is ready (described in the next paragraphs). Lots of early scanning work was consistent with the prediction that cerebellar processing, possibly linked to feedforward mechanisms, was implicated in stuttering (during normal speech and under fluencyenhancing conditions). Motor activity at the cerebellar level was considered a functional process (not a structural deficit) associated with regaining fluency control, where this operation was required to different extents by people who stutter and fluent speakers.

In the second step, it was proposed how execution receives inputs, programmes and executes them and checks that the plan for successive utterances was a complete one, how this changes over development and why this is reflected in changes in symptom type (stallings to advancings). Basically, an efferent copy of any speech issued for output is taken (Howell, 2002). It is subtracted from the plan after execution is completed. If the speaker started with the plan for a whole utterance, this was issued and there would be no difference. There would be a difference if the speaker started with an incomplete plan (as happens during advancing) because the incomplete plan is updated during the time for execution. In effect, the difference is not cancelled so a signal is generated that alerts the speaker that incomplete plans are being received and adjustment to speech timing is required. Importantly, the differencing mechanism is regarded as alerting the speaker that something went wrong, not anything specific about what went wrong (as in feedback accounts that determine that speech errors occurred).

This process would work in a person who persists in their stuttering as well as in fluent speakers during early development so the question arises about why the former speakers become less sensitive to the alerts. The proposed process by which this happens was simple physiological adaptation (Howell et al., 2000): By definition, speakers who continue to stutter would produce a lot of disfluencies that in turn lead to many alerts. Speakers who persist would ignore them, more would be produced, they would be adapted to and gradually the speakers who persist become less sensitive to the alerts. De facto, the alerting process ceases working in people who stutter.

\section{Neural substrates for atypical planning and execution of word production in stuttering}

The scanning experiment that Lu et al. (2010) performed examined planning and execution processes in speakers who stutter and controls. Examination of these joint processes is in line with recent thinking, reviewed above, that neither planning (cognitive-linguistic processing) nor execution (motor programming and output) are sufficient to account for stuttering (Howell, 2002; Howell, 2004; Howell, forthcoming). Instead, both planning and execution as well as the mechanism that interfaces them are necessary to explain stuttering. Lu et al.'s (2010) experiment involved isolated word production in which variables associated with planning and execution were manipulated independently. This was performed by groups of people who stutter and controls. The task variable associated with planning was "number of syllable sized motor programs". If the number of syllables in a 
word varies, its word length will change. Word length was controlled by having participants repeat a three-syllable word once or a one-syllable word three times. The task variable associated with execution was "syllable size and onset complexity". fMRI scans were taken at the time the task was performed. Comparison was made between the participant groups to determine what were the neural substrates of planning, execution and the interface between them and whether activation in these areas differed between the participant groups. The authors used the task-by-speaker group interactions to identify aberrant planning or execution processes in people who stutter.

Structural equation modeling was used to identify the neural substrates that were associated with each of the processes. The structures that were associated with planning were the bilateral inferior frontal gyrus and the right putamen; The structures that were associated with execution were the left premotor area and anterior gyrus, right insula, and the cerebellum; The structures that were associated with the interface were the left premotor area and anterior gyrus and the right insula.

\section{Locus of neural substrates associated with planning, execution and the interface}

EXPLAN only explicitly proposed what neural structures are associated with execution whereas Lu et al. (2010) also identified structures involved in planning and the interface. Of particular note in Lu et al.'s (2010) findings, is the fact that the cerebellum is involved in execution processes. A number of other scanning studies have also shown different cerebellar activity in people who stutter versus controls (Brown et al., 2005). The particular prediction EXPLAN makes about cerebellar involvement in stuttering was upheld by Lu et al.'s (2010) study.

\section{Structural and functional deficits at planning, execution and the interface}

According to the original version of EXPLAN, planning and execution processes could be functional problems although problems in planning that were due to structural factors were not ruled out. Subsequent evidence suggests that there is a structural planning problem in people who stutter. The most telling evidence is from two studies that have reported deficits in tasks that do not require speech output. These studies rule out motor artifacts which could lead to apparent differences in scanned images between speakers who stutter and controls leaving planning effects as the most likely alternative responsible for the differences. Biermann-Ruben et al. (2005) showed in a study using MEG that both the left inferior frontal and the right rolandic areas of stuttering speakers played similar roles in speech perception and production. Ingham et al. (2000) found similar types of neural differences between stuttering and non-stuttering speakers during imagined stuttering (i.e., speech planning without execution) and overt stuttering. These two studies suggest that stuttering speakers differ from non-stuttering speakers in their brain activations whether or not speech output is required (probably implicating planning processes).

It remains possible that functional differences occur in execution processes (as in the alerting account in EXPLAN) even if there is a structural planning deficit in people who stutter. Consistent with this, clinical evidence suggests degenerative disorders of the basal ganglia lead to development of stuttering in older patients that never had these symptoms as children. This suggests that structural damage to this area may result in stuttering and, conceivably, such damage may also account for developmental stuttering. In contrast, degenerative diseases of the cerebellum do not generally lead to stuttering and this may be because functional roles, like those performed in the cerebellum, can be done more flexibly and/or are less tied to specific tissue locations than are problems that arise from lesions in 
specific areas that are necessary for speech control such as planning. Subsequent work should aim to establish empirically whether activation in cerebellar-execution processes is functional or structural. Evidence is also needed about whether activation in the neural structures that operate as the interface is functional or structural and further evidence is also required about planning involving a structural deficit. Finally, better indications about whether functional cerebellar speech motor processes have more plasticity than structural language planning and interface processes are needed.

\section{Isolated words versus connected material}

As explained, EXPLAN is a model of connected discourse. In connected discourse planning and execution occur contiguously. Tasks in which objects in pictures are named allow speakers to plan before they start execution processes. In order to examine how the interface (in particular) operates, tasks with connected discourse are required.

\section{Recovery processes after fluency breakdown in people who stutter}

Imaging work in people who stutter has sought to identify what neural structures lead to the fluency failure in these speakers. EXPLAN maintains that there are also two distinct processes of recovery after such fluency failures (stallings and advancings) and that these should lead to activation in the different regions that have been identified by $\mathrm{Lu}$ et al. (those associated with the interface or execution). Linking activity in the regions identified with planning and execution to the symptom types associated with these processes (stalling and advancing respectively) is the next important step. Knowing whether the specified neural processes are involved in these forms of fluency failure may allow clinicians to monitor, and possibly prevent, changes that happen when a speaker who stutters is at risk of persisting in the disorder.

EXPLAN assumes that the plan is incomplete when either advancings or stallings occur, so no activation differences would be expected in the neural structures that are involved in planning for the two disfluency classes. During advancings, execution receives and acts on a faulty plan. As advancings were issued to execution, the neural structures at the interface received no alert and activity should be similar to what occurs when complete plans (fluent) are processed at the interface. When stallings occur, alerts are generated in the execution structures, the interface is disconnected, old plans are reprogrammed for motor output until a complete plan for the next word is received.

Sparse sampling techniques use the fact that the BOLD response continues after a speaker has stopped speaking and have been used to reduce motor artifacts in investigations into stuttering (Watkins et al., 2008). The intervals following either stallings or advancings could be grouped separately and these predictions about CNS activation for the different ways of recovering fluency could be examined for each class using a similar procedure to that employed by Braun et al. (1997) for different purposes. Thus, if the EXPLAN proposal about stalling/advancing recovery processes and the Lu et al. (2010) proposal about the sites responsible for planning, execution and the interface are correct, then activation after stallings and advancings should be equivalent at planning processes, but execution and interface areas should show different activations.

Further work should also be conducted to establish whether the proposed adaptation process operates differently in people who recover and persist. EXPLAN predicts that the speakers who persist are less sensitive to alerts generated at the execution level (in turn, the alerts would also be absent at the interface). Although different patterns of activity are predicted for persistent and recovered speakers at execution and interface sites, establishing whether they occur may be tricky because fluency severity effects need to be partialled out (the 
persistent group would be expected to have higher severity). Also, although persistent speakers are specifically expected to show adaptation, this may not result in lower activations in these speakers compared to recovered speakers. This is because adaptation may be a process that requires active inhibition (in which case more adaptation would be signified by higher activation levels).

\section{Acknowledgments}

This work was supported by grant 072639 from the Wellcome Trust to Peter Howell.

\section{Reference List}

Alm PA. Stuttering and the basal ganglia circuits: A critical review of possible relations. J Commun.Disord. 2004; 37:325-369. [PubMed: 15159193]

Biermann-Ruben K, Salmelin R, Schnitzler A. Right rolandic activation during speech perception in stutterers: A MEG study. Neuroimage. 2005; 25:793-801. [PubMed: 15808980]

Braun AR, Varga M, Stager S, Schulz G, Selbie S, Maisog JM, et al. Altered patterns of cerebral activity during speech and language production in developmental stuttering. An H2(15)O positron emission tomography study. Brain. 1997; 120(Pt 5):761-784. [PubMed: 9183248]

Brown S, Ingham RJ, Ingham JC, Laird AR, Fox PT. Stuttered and fluent speech production: An ALE meta-analysis of functional neuroimaging studies. Hum.Brain Mapp. 2005; 25:105-117. [PubMed: 15846815]

Dworzynski, K. Multilingual aspects of fluency disorders. Howell, P.; Van Borsel, J., editors. Multilingual Matters; Bristol, England: 2010. Forthcoming

Groszer M, Keays DA, Deacon RM, de Bono JP, Prasad-Mulcare S, Gaub S, et al. Impaired synaptic plasticity and motor learning in mice with a point mutation implicated in human speech deficits. Curr.Biol. 2008; 18:354-362. [PubMed: 18328704]

Haesler S, Rochefort C, Georgi B, Licznerski P, Osten P, Scharff C. Incomplete and inaccurate vocal imitation after knockdown of FoxP2 in songbird basal ganglia nucleus Area X. PLoS.Biol. 2007; 5:e321. [PubMed: 18052609]

Howell P, Rosen S, Hannigan G, Rustin L. Auditory backward-masking performance by children who stutter and its relation to dysfluency rate. Percept.Mot.Skills. 2000; 90:355-363. [PubMed: 10833723]

Howell P, Sackin S. Timing interference to speech in altered listening conditions. J.Acoust.Soc.Am. 2002; 111:2842-2852. [PubMed: 12083218]

Howell, P. Current issues in linguistic theory series: Pathology and therapy of speech disorders. Fava, E., editor. John Benjamins; Amsterdam: 2002. p. 95-118.

Howell, P.; Au-Yeung, J. Pathology and therapy of speech disorders. Fava, E., editor. John Benjamins; Amsterdam: 2002. p. 75-94.

Howell P. Assessment of some contemporary theories of stuttering that apply to spontaneous speech. Contemp.Issues Commun.Sci.Disord. 2004; 31:122-139. [PubMed: 18259590]

Howell P. A model of serial order problems in fluent, stuttered and agrammatic speech. Hum Mov Sci. 2007; 26:728-741. [PubMed: 17765346]

Howell P, Davis S, Williams R. Late childhood stuttering. J.Speech Lang Hear.Res. 2008; 51:669_ 687. [PubMed: 18506043]

Howell, P. Recovery from stuttering. Psychology Press; 2010. Forthcoming

Howell, P. The handbook on psycholinguistics and cognitive processes: Perspectives on communication disorders. Guendouzi, J.; Loncke, F.; Williams, M., editors. Taylor \& Francis; London: 2010. p. 437-464.

Ingham RJ, Fox PT, Costello IJ, Zamarripa F. Is overt stuttered speech a prerequisite for the neural activations associated with chronic developmental stuttering? Brain Lang. 2000; 75:163-194. [PubMed: 11049665]

Ivry R. Cerebellar timing systems. Int.Rev.Neurobiol. 1997; 41:555-573. [PubMed: 9378608] 
Johnson, W. The onset of stuttering. University of Minnesota Press; Minneapolis: 1959. Associates.

Kang C, Riazuddin S, Mundorff J, Krasnewich D, Friedman P, Mullikin JC, et al. Mutations in the lysosomal enzyme-targeting pathway and persistent stuttering. N.Engl.J Med. 2010; 362:677-685. [PubMed: 20147709]

Kawato M, Furukawa K, Suzuki R. A hierarchical neural-network model for control and learning of voluntary movement. Biol.Cybern. 1987; 57:169-185. [PubMed: 3676355]

Lan J, Song M, Pan C, Zhuang G, Wang Y, Ma W, et al. Association between dopaminergic genes (SLC6A3 and DRD2) and stuttering among Han Chinese. J Hum Genet. 2009; 54:457-460. [PubMed: 19590515]

Limber, J. Cognitive development and acquisition of language. Moore, T., editor. Academic Press; New York: 1973.

Lu C, Chen C, Ning N, Ding G, Guo T, Peng D, et al. The neural substrates for atypical planning and execution of word production in stuttering. Exp.Neurol. 2010; 221:146-156. [PubMed: 19879262]

Watkins KE, Smith SM, Davis S, Howell P. Structural and functional abnormalities of the motor system in developmental stuttering. Brain. 2008; 131:50-59. [PubMed: 17928317]

Wing AM, Kristofferson AB. Response delays and timing of discrete motor responses. Perception \& Psychophysics. 1973; 14:5-12.

Wingate ME. SLD is not stuttering. J.Speech Lang Hear.Res. 2001; 44:381-383. [PubMed: 11324659]

Yairi, E.; Ambrose, NG. Early childhood stuttering. Pro-Ed; Austin, Texas: 2005. 

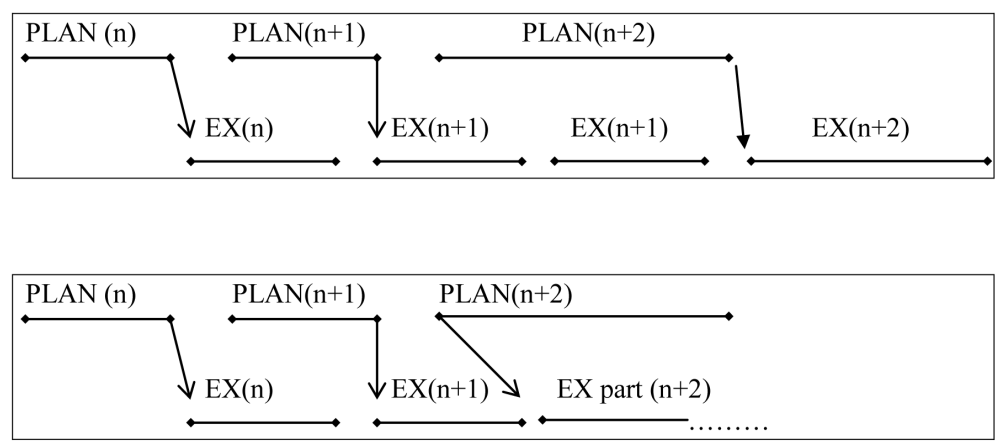

Figure 1.

Diagrammatic representation of the temporal relationship ( $\mathrm{x}$ axis) between planning and execution when a speaker stalls (top part) or advances (bottom part) according to the EXPLAN model. For both parts, planning of three successive words is shown in the top rows of each part (two short words to plan and one long word to plan, labeled word $n, n+1$ and $n+2)$. Execution of word $n$ commencing after its plan is complete as shown in the second rows of each part. While word $n$ is being executed, there is sufficient time to plan the following word $(n+1)$. Word $n+1$ is executed immediately after word $n$ has been completed and planning for word $n+2$ commences. At the point where execution of word $n+1$ is complete, the plan for word $n+2$ is not finished. In the top part for the case where the speaker stalls, the speaker repeats word $n+1$ and this allows sufficient time to complete the plan of word $n+2$ (that word can then be produced and the utterance continued). In the bottom part for the case where the speaker advances his or her speech (i.e. starts the utterance with an incomplete plan), the plan may run out in which case the speaker may sustain the first part of word $n+2$ (prolongation), repeat it (part-word repetition) or interrupt it (word break). 